\title{
A Robust Iris Identification System Based on Wavelet Packet Decomposition and Local Comparisons of the Extracted Signatures
}

\author{
Florence Rossant, Beata Mikovicova, Mathieu Adam, and Maria Trocan \\ LISITE (Laboratoire d'Informatique, Signal et Image, Electronique et Télécommunications), \\ Institut Supérieur d'Electronique de Paris (ISEP), 21 rue d'Assas, 75006 Paris, France \\ Correspondence should be addressed to Florence Rossant, florence.rossant@isep.fr
}

Received 1 December 2009; Revised 16 February 2010; Accepted 18 March 2010

Academic Editor: Yingzi Du

Copyright ( $\odot 2010$ Florence Rossant et al. This is an open access article distributed under the Creative Commons Attribution License, which permits unrestricted use, distribution, and reproduction in any medium, provided the original work is properly cited.

This paper presents a complete iris identification system including three main stages: iris segmentation, signature extraction, and signature comparison. An accurate and robust pupil and iris segmentation process, taking into account eyelid occlusions, is first detailed and evaluated. Then, an original wavelet-packet-based signature extraction method and a novel identification approach, based on the fusion of local distance measures, are proposed. Performance measurements validating the proposed iris signature and demonstrating the benefit of our local-based signature comparison are provided. Moreover, an exhaustive evaluation of robustness, with regards to the acquisition conditions, attests the high performances and the reliability of our system. Tests have been conducted on two different databases, the well-known CASIA database (V3) and our ISEP database. Finally, a comparison of the performances of our system with the published ones is given and discussed.

\section{Introduction}

Biometric systems provide reliable automatic recognition (identification) of persons based on one or several biological features. These systems are progressively replacing the conventional identification methods, such as documents, login passwords, or personal identification codes. There are several benefits of using biometrics in combination with or instead of traditional techniques. A first advantage is the ease of use: to be identified, a person does not have to remember a password or identification code and does not have to carry a key, and thus the identification process can be very quick. Second advantage, the protection of the identifier: a weak spot in many traditional security systems is that the users often write down their code or tend to choose a code which is easy to remember and thus also easy to break. Keys and cards can be stolen or copied. On the contrary, biometric systems can be made quite safe against forgery. Last but not least, the length of the code is a very important advantage: biometrics makes possible the use of very long codes and thus the bruteforce hacking strategies are inefficient.

Different types of biometrics such as fingerprints, hand geometry, facial appearance, voice, retina, and iris have been used. Nowadays, the iris is considered as one of the most reliable traits for biometric identification because of its random morphogenesis, great variability among different persons, and stability over time. The performances of iris based algorithms are better than those using other biometrics, as for example, the face recognition algorithms. However, iris recognition systems rely on good quality images and their performances deteriorate in unconstrained environments.

Iris based recognition systems have been widely studied for the last 20 years. It was in the early nineties that John Daugman implemented and patented an automated readyto-use iris recognition system $[1,2]$. Even though Daugman's system is the most successful and the most well known, many other approaches have been proposed. Typically, such recognition systems, in spite of their specificities, have the 
same structure: the first stage consists in the iris segmentation, then the image is normalized and features are extracted in order to generate a signature. Finally, this signature is compared to reference signatures (i.e., gallery database) in order to measure a numerical dissimilarity value to be used in the decision process.

The segmentation part, consisting of the localization and extraction of the iris, is crucial as the whole recognition system depends on its accuracy. Therefore, much research has been conducted on the segmentation, based essentially on two main methods: an integro-differential algorithm proposed by Daugman [2] and an algorithm based on the circular Hough transform employed by Wildes [3]. Many other methods have been proposed, combining these algorithms or employing some threshold-based methods [4] and, more recently, algorithms using active contour models [5]. An important part of the segmentation is the localization of occlusions caused by eyelids and eyelashes, hiding, at least partially, the iris texture. If they are not taken into account, they are considered as a part of the iris structure and lead to the deterioration of the performances. Methods used for the eyelids localization are similar to those used for the iris boundaries, mainly based on Daugman's and Wildes' method. The eyelashes segmentation methods are principally based on thresholding.

The normalization step transforms the iris region so that it has fixed dimensions, in order to allow comparisons. The dimensional differences are mainly due to variations in the pupil dimensions, varying imaging distance, rotation of the camera, head tilt, and ocular motion. The majority of systems use the transform proposed by Daugman [6] which translates the segmented iris into a fixed length and dimensionless polar coordinate system. Two other methods are worth of noting, the Boles' system [7] using virtual circles and Wildes' algorithm [3] employing an image registration technique.

The aim of the feature (signature) extraction is to provide the most discriminating information present in an iris pattern. The analysis of the iris is accomplished either globally on the whole iris or locally. The data (coefficients) issued from this analysis are then encoded to form a biometric signature. The feature representation approaches could be roughly divided into three major categories: phasebased methods (as proposed by Daugman in e.g. [1, 2]), zero-crossing representation methods $[7,8]$, and various texture-analysis-based methods [3, 9-12].

The identification step consists in the confrontation of the tested signature to those stored in a reference database (gallery). This comparison allows establishing their similarity (or dissimilarity, depending on algorithms). Then, a decision criterion has to be applied to classify correctly the user as being authentic or imposter. To compare signatures, different distance metrics are commonly applied. The Hamming distance is the most frequently used $[2,6,13,14]$. It can be modified to limit the comparison only to coefficients corresponding to nonoccluded part of the iris $[6,13,14]$ or weighted by local quality measures [15]. Other authors use Euclidean [8] or weighted Euclidean distance [16]. Wildes in [3] proposed a measure of normalized correlation between the acquired and the database signatures but used it only in the verification task.

The decision criterion used for classification is generally a simple threshold, obtained empirically on training databases. Some authors proposed other types of classifiers such as neural networks $[12,17]$.

In this paper, we propose a ready-to-use iris identification solution. We developed a complete reliable system with high performances that includes all steps of a classical iris identification scheme (Section 2). As explained in Sections 2.3, 2.4, and 2.5, we contributed to all these stages, either by proposing new methods or improving the existing approaches. We present a precise, accurate and robust segmentation method that takes into account the eyelid detection. An original method of signature extraction and comparison, based on a wavelet packet decomposition, is also presented. Section 3 describes a complete validation of the proposed method, realized on two different databases: CASIA (infrared) and ISEP (visible domain). We also evaluate the robustness of the algorithm with regards to acquisition conditions, by simulating changes in illumination, blurring, and optical axis deviation. The performances of the whole system are presented in Section 4 and compared to those described in the literature. Section 5 concludes the presented system and further improvements are suggested.

\section{Iris Identification System}

2.1. Iris Databases. The images used in this study were acquired in the visible domain (ISEP database) or in the near infrared domain (CASIA database). Both systems are cooperative, that is, the iris images were captured at small distances, under controlled lighting conditions and with cooperating subjects.

To create the ISEP database, we used a dedicated iris imaging equipment provided by Miles research lab [18]. It is made up of a Nikon camera with $105 \mathrm{~mm}$ lens. The flash illumination is precisely guided to the eye via fibre optics light-guides, in order to provide uniformly illuminated eye pictures with little light reflections (Figure 1(a)). Nevertheless, light spots are present in the pupil area and sometimes in the iris texture itself, when the flash is not perfectly centred. The images were resized to $600 \times 400$ pixels and transformed from RGB colour format to grey-levels, each pixel being coded on one byte.

The ISEP database contains 1572 images acquired from the left and/or the right eye of 337 different individuals. Most of them are European $(87.23 \%)$ but the database contains also images from African or Indian (10.1\%) or Asiatic $(2.67 \%)$ people. The acquisition protocol included two different acquisition conditions: in the first case, the eye was preilluminated to contract the pupil; in the second case, the picture was taken in indoor conditions, with no preillumination, providing images with largely dilated pupils. We have two images of both types for each of the 403 classes (i.e., eyes). 

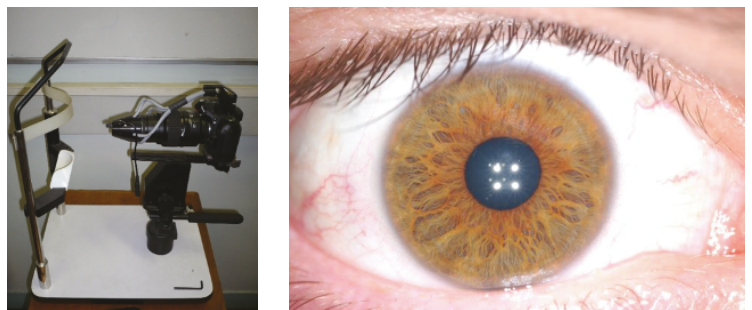

(a)
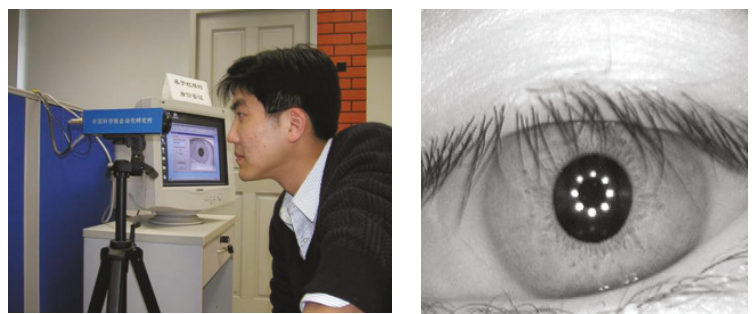

(b)

FIgure 1: Acquisition systems and iris images from (a) ISEP database and (b) CASIA database.

The CASIA-IrisV3-Interval is a public database provided by the Center for Biometrics and Security Research (CBSR) [19]. Iris images are 8 bit grey-level, $320 \times 280$ pixels, collected under near infrared illumination (Figure 1(b)). Almost all subjects are Chinese. Images extracted from CASIA database serve generally as references for the comparison of iris identification systems. In our study, we extracted a subdatabase including 888 images of 222 classes, having therefore 4 images per eye. The extraction process was conducted as follows: exclusion of images with very large occlusions (more than $50 \%$ of the iris texture) or inadequate segmentation (as explained in Section 2.3.4), exclusion of a class when less than four images are available under the previous conditions, and random selection of four remaining images per eye.

2.2. Functional Scheme. The functional scheme of the proposed system is depicted in Figure 2 and follows the typical structure of majority of iris recognition systems. The image is first segmented in order to extract the iris texture. Two major steps are required: the localization of the inner and outer iris boundaries (Sections 2.3.1 and 2.3.2) and the detection of the eyelids (Section 2.3.3). Our system provides a robust and accurate segmentation, which has been demonstrated by comparing the automatic segmentations with manual ones (Section 2.3.4). Then, the iris ring is unwrapped to get a rectangular image of normalized size. The information about the eyelid boundaries are used to define a binary mask, where pixels corresponding to iris texture are coded as 1's (Section 2.3.5). We propose then an original method for extracting signatures, based on a wavelet packet decomposition (Section 2.4). The identification part consists in the comparison between iris signatures. Two different approaches are proposed: a global comparison and a novel method based on a fusion of local distances calculated on subregions of the iris (Section 2.5).

\subsection{Segmentation and Normalisation. The segmentation step} aims at extracting the iris texture area from the eye image. Major difficulties come from the weak contrast between the pupil and the iris (dark eyes) or the iris and the sclera (pale eyes), but also from the poor quality of the acquired pictures. Images are indeed often defocused or occluded by eyelids or light spots. Nevertheless, efficient iris localization is required, since the whole recognition system depends on the accuracy of this segmentation step.

The proposed method is based on three steps: rough localization of the pupil, iris boundary detection, and eyelid detection. This way, the pixels belonging to iris texture are precisely determined. Further, the iris is unwrapped into a rectangular image of fixed size, using Daugman's polar transform [6]. A binary mask is also deduced from the eyelid segmentation results in order to distinguish precisely iris pixels from eyelid pixels.

\subsubsection{Rough Detection of the Pupil. This step can be, to a} certain extent, dependent on the illumination system used for image acquisition. Nevertheless, the proposed method is robust enough to process both CASIA (Figure 3(a)) and ISEP databases. The bright spots are detected based on top-hat morphological filters. They are then removed by a specific filter which replaces every spot-pixel (i.e., pixels contained within a bright spot) by an average of its nearby pixels, starting from the spot periphery and progressing iteratively towards the centre (Figure 3(b)). The average includes only pixels that were not labelled as spot-pixels beforehand or those which have been processed at a previous iteration. So, the spots are filled with dark pixels without creating high gradients.

In the following, we assume that the pupil is almost centred in the image (cooperative acquisition system) and define a region of interest $(160 \times 160$ pixels $)$ in order to localize it roughly (Figure 3(b)). This area is represented in a binary manner, for selecting the darkest pixels that are likely to belong to the pupil (Figure 3(c)). The threshold is dynamically adjusted through the histogram analysis, in order to keep the $15 \%$ of darkest pixels. Morphological filters are applied to improve the segmentation quality and the region of largest area is kept as pupil region. The pupil centre is estimated from the bounding box and the gravity centre of the extracted region (Figure 3(d)).

2.3.2. Iris Boundary Localization. This second step consists in the localization of the inner and outer iris boundaries, considered either circular or elliptical. Two major methods were proposed by Daugman and Wildes. Both are based on the use of the first derivatives of the image and a circular parametric model of the iris contours. The best parameters 


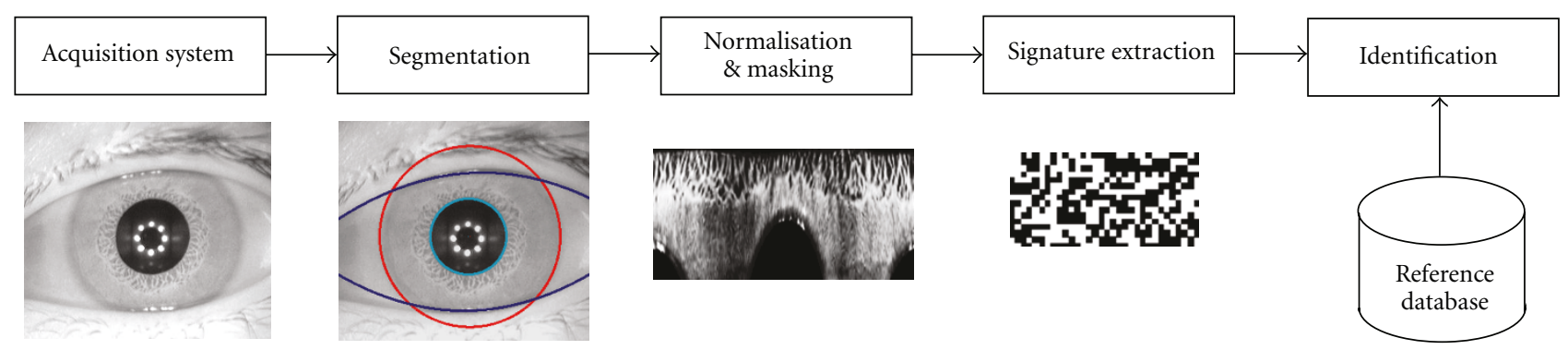

Figure 2: Functional scheme of the complete recognition system.

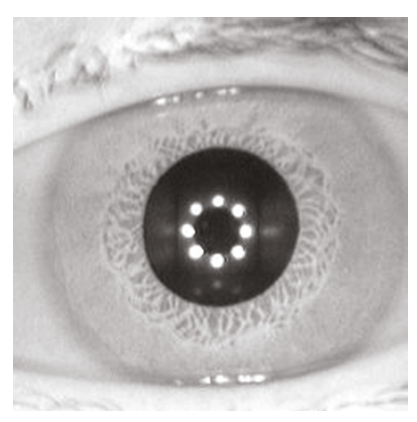

(a)

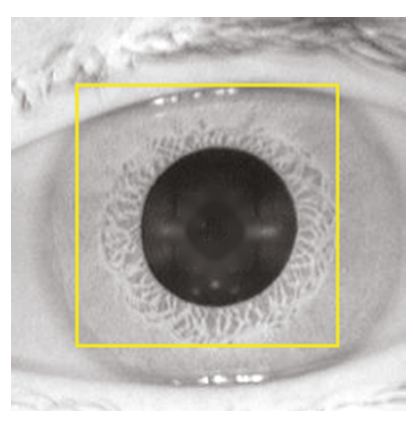

(b)

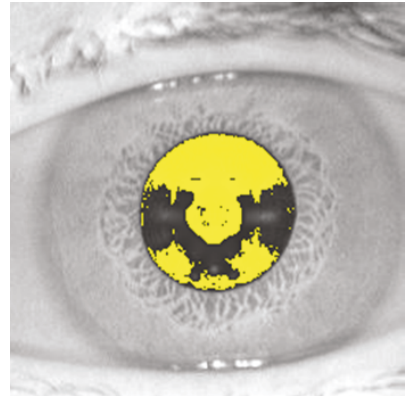

(c)

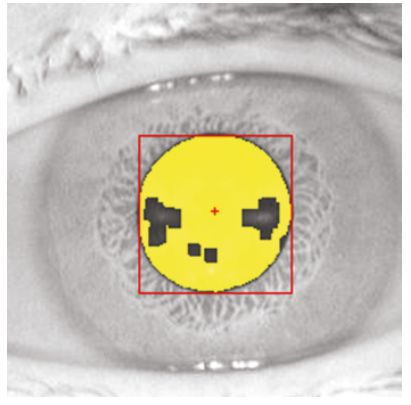

(d)

Figure 3: Rough localization of the pupil. (a) Source image, (b) filtered image and definition of the zone of interest for the pupil detection, (c) pupil detection by thresholding means, and (d) pupil localization (bounding box and centre estimation).

are obtained either by maximizing the output of an integrodifferential operator [2] or by using a Hough transform applied on a binary edge map [3]. The second method is probably less accurate than the first one since it depends on a threshold to be chosen for the edge detection and, thus sensitive to different types of images and illumination conditions. Moreover, the results provided by the Hough transform are often sensitive to the sampling of the space parameters.

Our method is similar to Daugman's one, but with a circular model of the pupil boundary and an elliptical model of the outer contour of the iris. The centre of the pupil and the centre of the iris are supposed to be close to one another. From the first estimation of the pupil location (Figure 3(d)), we deduce a grid of possible coordinates for the pupil centre. Then, we find the centre and the radius of the circle that maximize the mean gradient in the orthogonal direction of the circular curve (Figure 4(a)). The gradient is estimated by a correlation with the $1 \mathrm{D}$ kernel $[-1 \quad-$ $10+1+1]$, representing an ideal step. This process is applied on the preprocessed image to avoid high gradients due to illumination spots. A similar algorithm is used for determining the ellipse, the possible centres being restricted in the neighbourhood of the pupil centre. However in this case, the gradient maximization is limited to the left and right subparts of the ellipse in order to avoid possible eyelid or eyelash occlusions (Figure 4(b)). This algorithm provides very good results (Figure 4(c)), even in case of very low contrast, because of the average effect, and because there is no need of parameter tuning as we use a maximization criterion.
2.3.3. Eyelids Localization. The detection of eyelid occlusions is crucial to achieve good identification rates. Most proposed methods are based on the analysis of the gradient image, assuming a high gradient at the frontier between the eyelid and the iris. But the selection of relevant contours is a very difficult task since eyelashes often hide eyelid boundaries and highly textured iris also provides high gradients. That is why a priori knowledge about the shape of the searched contour is again required. Therefore most authors model eyelids as parabolic $[3,6,15]$ or circular arcs, and apply again an integro-differential operator [6] or a Hough transform [3] to select the best parameters of the parametric representation. Additional criteria are sometimes added to shortlist admissible contours: eyelashes detection, since they are supposed to be at the border of the eyelid [20], which requires an additional difficult step; selection of the longest edge [15], but the searched contours are often cut by eyelashes; denoising based on a statistical model that need to be learnt beforehand [21].

To improve the robustness of the process, we propose an algorithm [22] based on three steps: preprocessing, preselection of edge candidates to eyelids including first approximation and, finally, a decision by optimization of the mean gradient. In the preprocessing stage, a nonlinear diffusion filter is applied to smooth the iris texture, while sufficiently preserving the eyelid boundaries (Figure 5(a)). The second step consists in applying a Canny-edge detector in order to obtain a map of edge candidates. A priori knowledge about the position of the eyelids, with respect to the iris boundaries, is used to perform a first selection of 


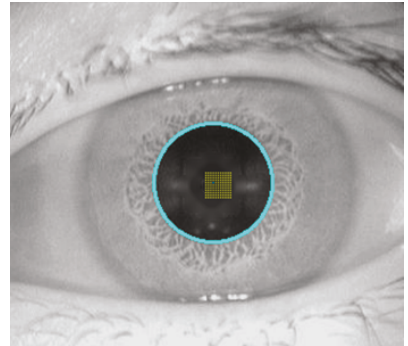

(a)

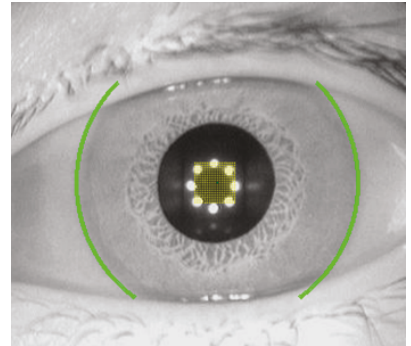

(b)

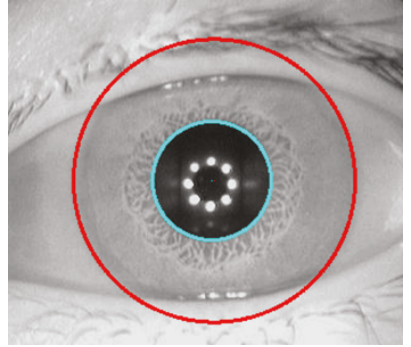

(c)

FIGURE 4: Iris boundary localization. (a) Estimation of the pupil contour by a circle (in yellow, the grid of the tested centres), (b) estimation of the outer iris contour by an ellipse, and (c) obtained result.

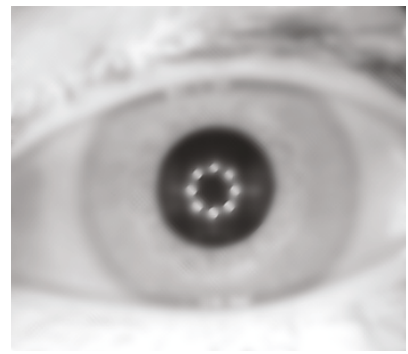

(a)

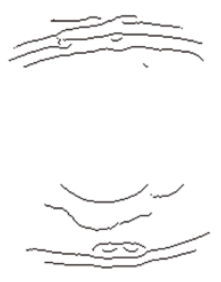

(b)

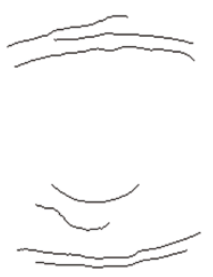

(c)

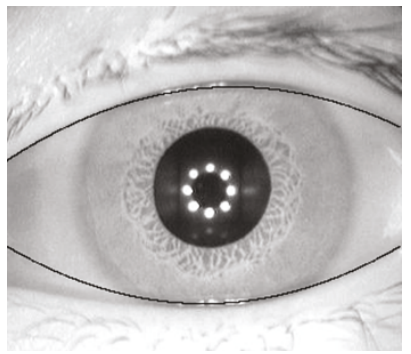

(d)

Figure 5: Occlusion localization. (a) Preprocessed image, (b) selected edges to be fitted by a parabola,(c) final candidate selection, and (d) edge detection after optimization.

the relevant edges. Therefore, we restrict the analysis area to the inner iris. Afterwards, we remove the left and the right parts of the iris in order to avoid connections between the eyelids/iris and the iris/sclera boundaries. We thus keep the edges that are the most likely to belong to the eyelid border (Figure 5(b)), so that the speed and the robustness of the algorithm are improved. The remaining edges, whose by length is greater than the mean, are fitted by a parabolic curve. More side-knowledge is introduced by eliminating the parabolas which have inaccurate orientation. At this stage, only 2 to 30 edge candidates still remain (Figure 5(c)). In the third step, the analysis is refined: the mean gradient along each candidate is calculated for different values of the parabola parameters around the first estimation, on a larger area (twice the size of the iris in the horizontal direction). The mean gradient is estimated on the result of the horizontal Sobel kernel filtering of the original image, in order to focus on the horizontal edges. A global maximisation is then performed to select the parabola approximating the eyelid/iris frontier (Figure 5(d)).

2.3.4. Performance Evaluation of the Segmentation Process. The automatic segmentations were compared with segmentations realized manually on images from CASIA V3Interval database (2655 images) and ISEP database (1572 images), in order to provide a quantitative evaluation of the segmentation process. Let us denote by $\left(x_{p}^{(A)}, y_{p}^{(A)}\right)$ and $r_{p}^{A}$ the centre and radius of the pupil obtained with the proposed method, and by $\left(x_{p}^{(M)}, y_{p}^{(M)}\right)$ and $r_{p}^{M}$ the parameters obtained manually, serving as reference. We compute the relative error made on the pupil centre estimation by

$$
E_{\text {pupil_centre }}=\frac{\sqrt{\left(x_{p}^{(A)}-x_{p}^{(M)}\right)^{2}+\left(y_{p}^{(A)}-y_{p}^{(M)}\right)^{2}}}{r_{p}^{(M)}}
$$

and on the radius estimation by

$$
E_{\text {pupil_radius }}=\frac{\left|r_{p}^{(A)}-r_{p}^{(M)}\right|}{r_{p}^{(M)}} .
$$

The relative errors commited on the outer iris boundary localization are calculated in the same way, replacing $r_{p}^{M}$ by the horizontal axis of the ellipse in (1), and by the horizontal or vertical axis of the ellipse in (2). Table 1 indicates the percentage of images with a relative error of less than $5 \%$ and $10 \%$, respectively. The correspondence between the percentage and the number of pixels (in average), is also provided.

As expected, the presented results demonstrate better performances for the detection of the pupil on images illuminated with near-infrared light (CASIA database) rather than images illuminated with visible light (ISEP database), since the iris/pupil contrast is higher in the first case. On the opposite, the determination of the iris centre and of the 
TABLE 1: Evaluation of the accuracy of the segmentation process.

\begin{tabular}{|c|c|c|c|c|c|c|c|}
\hline & & \multicolumn{3}{|c|}{ ISEP } & \multicolumn{3}{|c|}{ CASIA } \\
\hline & & $<5 \%$ & $<10 \%$ & $\% \leftrightarrow$ pixels & $<5 \%$ & $<10 \%$ & $\% \leftrightarrow$ pixels \\
\hline \multirow{2}{*}{ Pupil } & Centre $\left(E_{\text {pupil_centre }}\right)$ & $77.4 \%$ & $94.8 \%$ & $1 \% \leftrightarrow 0.53 \mathrm{px}$ & $92.8 \%$ & $95.1 \%$ & $1 \% \leftrightarrow 0.42 \mathrm{px}$ \\
\hline & $\operatorname{Radius}\left(E_{\text {pupil_radius }}\right)$ & $86.1 \%$ & $95.6 \%$ & $1 \% \leftrightarrow 0.53 \mathrm{px}$ & $95 \%$ & $96 \%$ & $1 \% \leftrightarrow 0.42 \mathrm{px}$ \\
\hline \multirow{3}{*}{ Iris } & Centre & $92.5 \%$ & $99.2 \%$ & $1 \% \leftrightarrow 1.4 \mathrm{px}$ & $81.9 \%$ & $94.2 \%$ & $1 \% \leftrightarrow 1.1 \mathrm{px}$ \\
\hline & Horizontal axis & $95 \%$ & $99.6 \%$ & $1 \% \leftrightarrow 1.4 \mathrm{px}$ & $92.2 \%$ & $97.8 \%$ & $1 \% \leftrightarrow 1.1 \mathrm{px}$ \\
\hline & Vertical axis & $57.3 \%$ & $95.2 \%$ & $1 \% \leftrightarrow 1.4 \mathrm{px}$ & $64.1 \%$ & $92.4 \%$ & $1 \% \leftrightarrow 1 \mathrm{px}$ \\
\hline
\end{tabular}

horizontal axis parameter of the ellipse is more accurate for the ISEP database than for the CASIA database. Nevertheless, the vertical axis parameters are less accurate than the horizontal ones, for both databases. Indeed, the detection is disturbed by the presence of eyelids and eyelashes.

It should be noticed that the real position of the pupil centre was out of the grid of possible centres, determined through the rough location of the pupil (Section 2.3.1 and Figure $4(\mathrm{a})$ ), for only $0.4 \%$ of the ISEP images and $0.97 \%$ of the CASIA images. This demonstrates the robustness of this first segmentation step. However, the overall segmentation performances might still be improved by enlarging the grid.

These results are however difficult to compare with the ones given in the literature, since the databases are different (CASIA V1 in the literature) and no quantitative criterion has ever been expressed, to our knowledge.

A complete evaluation of the eyelid detection was also realized on the whole CASIA-IrisV3-Interval database (2655 images) [22]. The automatic segmentations were compared with manual segmentations. We defined the global error as the percentage of subsegmentation or oversegmentation. For the lower eyelids, we obtain $97.6 \%$ of localizations with less than $10 \%$ of global error. For the upper eyelids, $87.5 \%$ of localizations have less than $10 \%$ of global error. We also noticed that the system is very robust to oversegmentation. As stated above, these performance rates cannot be compared to those given in the literature, since the authors provided only qualitative assessment and on a different database (CASIA V1).

Figure 6 summarizes the segmentation performances obtained on all available images, including those presenting erroneous detection of iris borders. This graphic represents the proportion of images whose global error is lower than a given value, where the global error is computed as

$$
\begin{aligned}
& \text { Error }_{\text {Global }} \\
& =100 \frac{\text { number of sub or oversegmented pixels }}{\text { number of nonoccluded iris pixels }} .
\end{aligned}
$$

Approximately $90 \%$ of the images have a global error less than $10 \%$ for both databases, which demonstrates the reliability of the segmentation process. However, it should be pointed out that the different sources of inaccuracy do not have the same influence on the system identification rates. As underlined in [23], the pupil centre position is the most critical parameter, since it serves as origin of the normalization process (Section 2.3.5), while recognition systems are more tolerant to inaccuracies related to the

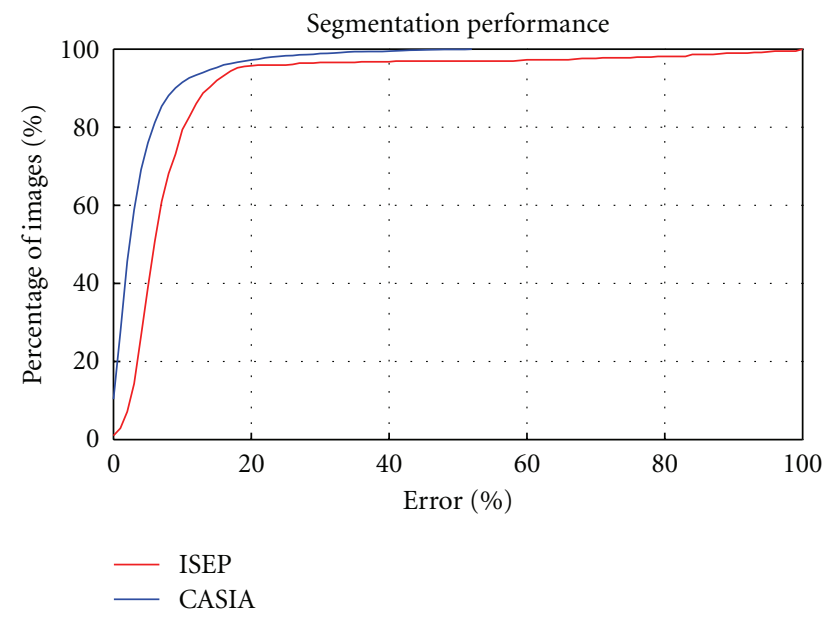

FIGURE 6: Global segmentation performance for the considered databases.

iris/sclera border. Moreover, imprecision on the eyelids detection are much less critical, since it has no influence on the normalization process. This fact was clearly demonstrated by experiments conducted on the CASIA database [24]. Indeed, the identification error rates are zero on the manually segmented CASIA database, when segmenting the eyelids, and are only slightly degraded when not considering the occlusions. On the opposite, the identification error rates come to $1.80 \%$ in middle security mode and to $5.43 \%$ in high-security mode, when testing the fully automatic system including the proposed segmentation step.

In what follows, we use only images that are correctly segmented, those for which the global error is less than $25 \%$ (thus, less than $2 \%$ of image rejection).

2.3.5. Normalization. The normalization stage is usually accomplished by using the method proposed by Daugman [2]. This model remaps each point within the iris region to a pair of polar coordinates $(r, \theta)$ where $r$ is on the interval $[0,1]$ and $\theta$ is an angle on the circle $([0,2 \pi])$. The angle sampling step is predefined, as well as the number of pixels sampled along each radius, so that the output image is a rectangle of fixed size. Let us denote by $n_{\theta}$ and $n_{r}$ the number of points along the angle and the radius axes. We designate the coordinates of pairs of pixels located on the pupil and the iris borders, aligned with the pupil centre and forming an angle $\theta_{n}$ with the $x$-axis, as $\left(x_{p}\left(\theta_{n}\right), y_{p}\left(\theta_{n}\right)\right)$ 


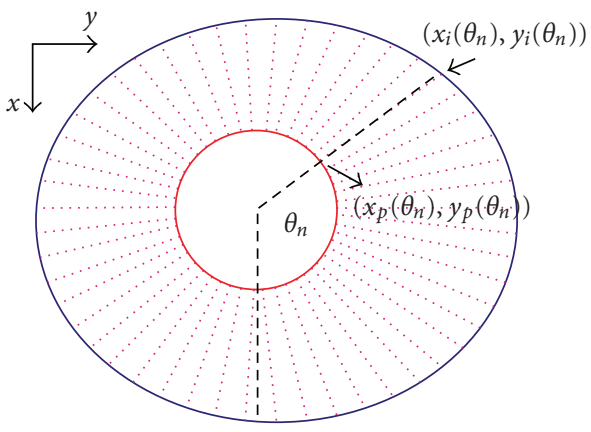

Figure 7: Polar transform.

and $\left(x_{i}\left(\theta_{n}\right), y_{i}\left(\theta_{n}\right)\right)$, respectively, (Figure 7). The Daugman's transform is expressed as

$$
\left\{\begin{array}{l}
x\left(r_{k}, \theta_{n}\right)=r_{k} x_{i}\left(\theta_{n}\right)+\left(1-r_{k}\right) x_{p}\left(\theta_{n}\right), \\
y\left(r_{k}, \theta_{n}\right)=r_{k} y_{i}\left(\theta_{n}\right)+\left(1-r_{k}\right) y_{p}\left(\theta_{n}\right),
\end{array}\right.
$$

with

$$
\begin{cases}\theta_{n}=\frac{n}{n_{\theta}} 2 \pi, & n=0, \ldots, n_{\theta}-1, \\ r_{k}=\frac{k}{n_{r}-1}, & k=0, \ldots, n_{r}-1 .\end{cases}
$$

Even though other normalizations have been proposed (e.g., [3, 7]), Daugman's transformation is commonly adopted since it easily deals with pupil dilatation or focal variations. Moreover, as the signatures are computed from the normalized images, no further normalization is necessary to compare signatures.

The size of the unwrapped images is set to $n_{\theta} \times n_{r}=$ $256 \times 128$ pixels (Figure $8(\mathrm{a})$ ). The binary mask defining the pixels belonging to the iris texture is defined in the same way (Figure 8(b)). The last step consists in a histogram equalization that increases the contrast of the texture and normalizes dynamically the grey-levels (Figure 8(c)).

2.4. Signature Extraction. Finding the appropriate features for the description of the unwrapped iris images represents the key for a robust signature extraction and classification. The literature acknowledges manifold of propositions. For example, Daugman $[2,6,13,14]$ applied 2D Gabor filters for extracting the phase structure information of the iris. Boles and Boashash [7] have chosen zero-crossing representations of the 1D wavelet transform of a concentric circle on the iris image, at various resolution levels, in order to characterize the iris texture. Wildes [3] decomposed the iris region using LoG (Laplacian of Gaussian) filters. The resulted filtered image is thus represented as a 4-level Laplacian pyramid and further used for generating a compact iris signature. Lim et al. [12] decorrelated the iris images using a 4-level 2D Haar transform and quantized the high-frequency information thus obtained. Ma et al. [9-11] defined new spatial filters to capture local details of the iris. Tisse et al. [25] introduced a concept of instantaneous phase and/or emergent-frequency.
The instantaneous phase is obtained by constructing an analytic image which is a combination of the original image and its Hilbert transform. Module of emergent frequency and the real and the imaginary parts of the instantaneous phase are used to encode the iris texture.

Lately, the subband decomposition methods [26] have gained a lot of interest due to their demonstrated efficiency in characterising different types of textures. Among these schemes, some have employed separable wavelet basis [7, $12,15,27$, as well as wavelet packet basis [28] in order to represent the analysed texture in a way that discriminant features are highlighted. A major inconvenience of the wavelet representation, however, is that only a subset of the possible space-frequency segmentation is used for the extraction of the spatial frequency components of the texture. Wavelet packets (WP) provide a solution to this problem so that full or adaptive frequency segmentation for a given texture can be obtained.

Especially for images with highly textured content, or residual textures (as the unwrapped iris images), the energy compactness performance of the wavelet packet subband structures is superior to classical wavelet one, as it has been shown in [29]. Moreover, a valid reason for using WP for iris signature extraction is that cyclic events (e.g., unwrapped iris strips) produce regular patterns in the spatial domain which can be efficiently represented by wavelet packet means.

As the WP transform [30-32] generalizes the dyadic wavelet decomposition by iterating the decompositions on the high-pass bands, it can be implemented by using a pair of Quadrature Mirror Filter (QMF) banks that divide the frequency bands into equal parts. This recursive splitting of the vector space is represented by the admissible WP tree (Figure 9).

There are two major categories of features extraction methods employing wavelet packets. The first one uses abstract aggregates of the original wavelet packet features such as: entropy, energy, distance, and so forth. on the full WP decomposition tree $[33,34]$. The second category clusters the best-basis WP feature extraction methods. Generally, in this latter class, the WP decomposition coefficients are used to form a feature space by merging specific nodes of the WP tree and splitting others, in order to produce a tree that represent the best reflection of the properties of the texture $[29,33]$. The features are then extracted based on some criteria applied to the wavelet coefficients in the terminal nodes of the resulted optimized tree [35].

Therefore, a compact iris signature can be obtained by quantizing the coefficients of the full WP decomposition tree into one bit each, depending on their sign [34]. In the following we propose a signature extraction method (which could be classified as belonging to the first category) which uses the energy of the WP coefficients as discriminator for determining which subbands carry the most useful part of the information.

The subbands to be analysed will be generated by the full 3-level WP decomposition using the orthogonal Haar transform, as shown in Figure 9. Due to the orthogonality of the involved transform, the energy preserving criterion is fulfilled. We can thus use the normalized WP subbands 


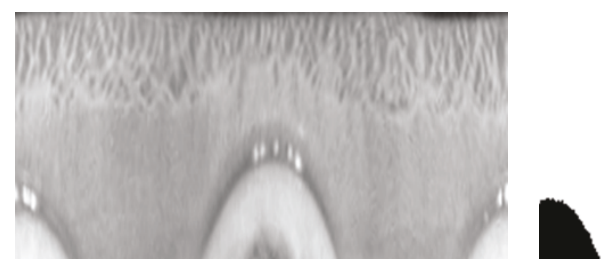

(a)

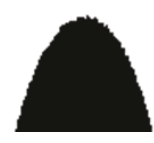

(b)

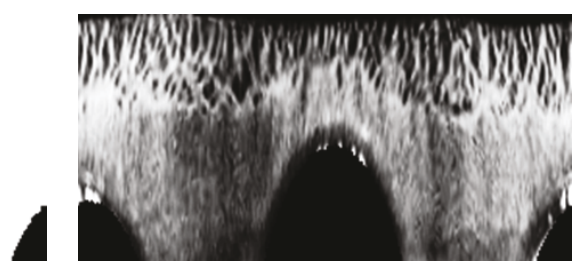

(c)

FIGURE 8: Normalization process. (a) Unwrapped image, (b) binary unwrapped mask, and (c) equalized iris texture with masked occlusions.

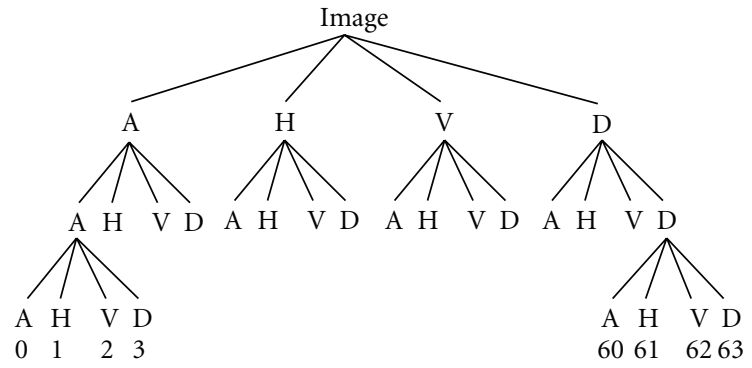

Figure 9: Full 3-level wavelet packet decomposition tree: the lowpass subbands (approximations) are denoted by A and the high-pass ones are represented following their directions- $\mathrm{H}$ (horizontal), $\mathrm{V}$ (vertical), and $\mathrm{D}$ (diagonal).

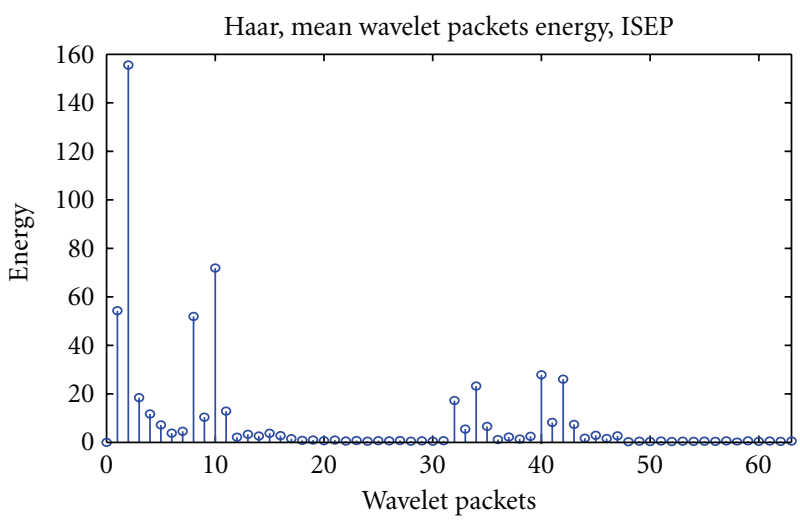

FIgURE 10: Mean wavelet-packet energy distribution.

energies $E_{i=0, \ldots, 63}$ as discriminator for deciding which packets should be considered for signature extraction

$$
E_{i}=\frac{1}{N_{i}} \sum_{j, k} w_{i}^{2}(j, k),
$$

where $w_{i}(j, k)$ denotes the $i$ th subband wavelet packet coefficients $\left(N_{i}\right.$ gives the total number of coefficients of the $i$ th subband).

As shown in Figure 10, the subbands 2 (AAV) and 10 (AVV) are efficient discriminants for the mean-energy value criterion. We propose thus to select these two most energetic subbands of the full WP decomposition tree and generate the signature bitwise as in the following:

$$
\begin{aligned}
s_{i}(j, k) & =\operatorname{sign}\left(w_{i}(j, k)\right), \quad \text { if }\left|w_{i}(j, k)\right|>T, \\
& =0, \quad \text { otherwise, }
\end{aligned}
$$

where the threshold $T$ is dynamically computed, following the descending magnitude order of the absolute values of the coefficients, such that only the most relevant ones retaining a certain percentage (Section 3.1) of the subband energy are sign-quantized. This way, similar to the wavelet-based denoising algorithms, the small coefficients are thus assimilated to noise and therefore filtered, in order to decrease their impact in the signature matching process. We thus obtain for each of the two energy-selected packets a robust signature, each represented by $16 \times 32$ symbols $(\{-1,0,1\})$ and which will be coded on 384 bytes.

The choice of the orthogonal Haar transform and of the quantization method has also been retrospectively validated by experiments (see Section 3.1).

2.5. Identification Method. The final task to be performed in an iris recognition system is the iris matching, that is, the comparison of the tested iris signature to signatures stored in a reference database, followed by the decision classifying the iris as an authentic or impostor. As mentioned before (see Section 1), different distance metrics have been proposed (Hamming, Euclidean, Manhattan) as well as different classifiers (from simple thresholds to neural networks classifiers).

In [36], we proposed a global iris identification method based on a normalized Manhattan distance measure. The applied measure combines the normalized Manhattan metrics given by signatures extracted from two wavelet packets (subbands 2 and 10). Two signatures for each individual are stored in the reference database. The tested signature is compared to both signatures of each individual and the minimum distance is retained to provide the final measure of dissimilarity. The eyelid occlusions are also taken into account [24].

However, this kind of global analysis cannot deal with distortions due to segmentation imprecision. Indeed, many parameters, related to the image acquisition process, impact the segmentation results and are responsible for the segmentation inaccuracies observed in Section 2.3.4. Especially, the optical axis, the illumination conditions, the pupil size, and the partial occlusions by eyelids or eyelashes are causes of segmentation variability: displacement of the 


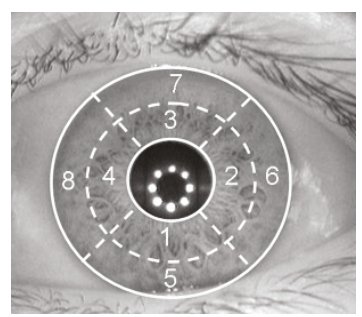

(a)

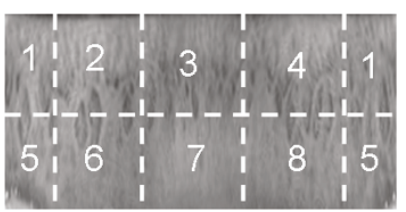

(b)
Figure 11: (a) The 8 areas in the source image, (b) corresponding to 8 rectangular blocks in the unwrapped image $(128 \times 256)$.

circle or ellipse centre, change of the radius or ellipse axis parameters. This variability results in translations of the iris structures in the unwrapped image, as well as dilation or contraction, especially in the radial direction, thus disturbing the signature comparison step. Consequently, despite the demonstrated robustness of the segmentation process (Section 2.3.4), acquisition conditions lead to iris detection inaccuracies that strongly affect the identification performances $[23,24]$.

To cope with such local distortions due to segmentation imprecision, we propose a novel signature comparison method based on a fusion of local distances. The reason is that the local distortions are not uniform over the unwrapped image. Another idea is to give more importance to iris areas which are likely to provide more reliable information. In our method, the iris is divided in eight subregions, equivalent to eight rectangular subwindows in the unwrapped image, as illustrated in Figure 11.

Blocks 1 to 4 correspond to the texture close to the pupil. As blocks 5 to 7 are related to peripheral textures, they are more prone to occlusions.

2.5.1. Local Distance Measures. The first step of our identification method consists in a global angular shifting to compensate for eye rotation between the reference and the tested image [34]. Local comparisons are then performed, as described in what follows.

The tested unwrapped image is extended by $N$ pixels in both directions to authorize horizontal and vertical sliding of the subwindows with minimized side effects. $N$ is the maximum shift, in pixels, of the subwindows.

The comparison is therefore independently realized on each rectangular block of the unwrapped image. The blocks of the tested unwrapped image slide along the vertical and horizontal directions, around their central position, while the subwindows of the reference image are fixed.

Let us denote by $S_{i,\{m, n\}}^{T, b}(j, k)$ the coefficients of the signature derived from packet $P_{i}(i=2,10)$, for the subwindow $b(b \in[1,8])$ of the tested iris $T$ and the translation $\{m, n\}(m \in[-N, N])$. We denote by $S_{i}^{R, b}(j, k)$ its equivalent for the reference iris $R \cdot M_{\{m, n\}}^{T, b}(j, k)$ are the binary masks defining the nonoccluded coefficients (corresponding to iris texture) for the subwindow $b$ and the translation $\{m, n\}$ of the tested iris $T$, and $M^{R, b}(j, k)$ their equivalent for the reference iris $R$ [22]. The distances $\operatorname{HD}_{i,\{m, n\}}^{b}$ between the tested and reference subsignatures derived from packet $P_{i}$ for the subwindow $b$ and the translation $\{m, n\}$ are computed as follows:

$$
\begin{aligned}
& \operatorname{HD}_{i,\{m, n\}}^{b} \\
& =\frac{1}{2 N_{b}} \sum_{j, k}\left|S_{i,\{m, n\}}^{T, b}(j, k)-S_{i}^{R, b}(j, k)\right| M_{\{m, n\}}^{T, b}(j, k) M^{R, b}(j, k),
\end{aligned}
$$

where $N_{b}$ is the number of coefficients equal to 1 in both masks for the subwindow $b$.

The local distances measured for the subwindow $b$ and combining two wavelet packets are obtained as

$$
D_{\{m, n\}}^{b}=2 \mathrm{HD}_{2,\{m, n\}}^{b} \mathrm{HD}_{10,\{m, n\}}^{b} .
$$

As each considered subband provides specific discriminating information, the use of the product as fusion rule is very pertinent, increasing the discrimination power of the classifier. Other conjunctive rules, such as the minimum, would provide less selective results.

Subsignatures are generated for all the $(2 N+1) \times$ $(2 N+1)$ possible block translations and compared to the corresponding reference subsignatures. This process leads to a set of $(2 N+1) \times(2 N+1)$ distance measures for each block. The optimal superposition between the tested and the reference subsignatures is given by the minimum distance $D^{b}$ :

$$
D^{b}=\min _{m, n} D_{\{m, n\}}^{b}, \quad b \in[1,8] .
$$

In this way, eight distances are obtained, each corresponding to the analysis of a subwindow. The aim of the next step is the fusion of these local distances, in order to get a final measure representing the global dissimilarity of both irises.

2.5.2. Fusion of Local Distances. In the literature, diverse fusion rules are admitted, such as the minimum or maximum, the arithmetical or geometrical average [37]. In our system, we take into account some additional knowledge by giving more importance to the local distances corresponding to the most informative and reliable areas. Therefore, we choose a weighted sum as fusion rule and the global distance between the tested and the reference iris is given by:

$$
D_{w}=\sum_{b=1}^{8} w^{b} D^{b} .
$$

In this equation, the weight $w^{b}$ is a combination of two weights, in order to take into account knowledge about the information quantity carried by the different subregions and their reliability. The first weight, denoted by $w_{d}^{b}$, represents the proportion of coefficients corresponding to nonoccluded iris texture.

The second weight, $w_{s}^{b}$ allows giving less importance to the local measures that are statistically different from the others. Thus, the influence of blocks that are likely to be 
unreliable or nondiscriminating is reduced [24]. Let $m$ be the mean and $\sigma$ the standard deviation of the eight local distances $D^{b}$ obtained between the tested iris and a reference iris (9). The weight, denoted as $w_{s}^{b}$, is given by the following equation:

$$
w_{s}^{b}= \begin{cases}p, & \text { if } D^{b}<m-\sigma \\ p, & \text { if } D^{b}>m+\sigma \\ 1, & \text { otherwise, }\end{cases}
$$

where $p$ is less than 1 .

The final weight of block $b$ is defined by:

$$
w^{b}=\frac{w_{d}^{b} w_{s}^{b}}{\sum_{k=1}^{8} w_{d}^{k} w_{s}^{k}}
$$

In our algorithm, two parameters are required: the maximum shift $N$ and the parameter $p$ of the weight $w_{s}^{b}$. Both are obtained in a prior training phase on a representative subset of the database. The optimal values of parameters are those which minimize the error rates: $N=3$ and $p=0.8$. These learnt parameters are then validated on a test set that is independent of the training set [24].

In what follows, we will refer to the measure derived from (10), (11), and (12) with $N=0$ and $p=1$ as global comparison. In this case, the measure is made globally on the nonoccluded iris texture.

As experiments show (see Sections 3 and 4), significant improvement of performances is obtained when applying the local identification approach instead of the global one.

\section{Validation and Performances Evaluation}

In this Section, we propose the simulation framework for both CASIA and ISEP databases, in order to validate parameters involved in the algorithm and to evaluate the robustness of the proposed method. In our experiments, we have considered 888 images of 222 classes from each of our databases. The retained ISEP images were selected randomly. Indeed, the performances measurements should not be biased by different numbers of classes, in order to be comparable.

In the following, we express the performance results in terms of false reject rate (FRR), false accept rate (FAR) and equal error rate (EER). The FAR measures the rate of impostors accepted by the system, the FRR measures the rate of authentics rejected by the system, and the EER is the rate where FAR = FRR. Moreover, we study the error rate in highsecurity mode: the FRR when preventing the false accept errors (denoted by FRR (FAR $=0 \%)$ in what follows).

3.1. Signature Extraction. The energy of the WP coefficients is used as discriminator for determining which subbands carry the most useful part of the information for the signature generation, as explained in Section 2.4. The study was first made using the Haar wavelet, but other wavelets could be suitable for generating the signature. Especially, the Biorthogonal 1.3 leads to a similar energy repartition of
TABLE 2: EER and FRR (FAR $=0 \%$ ) measured on (a) CASIA and (b) ISEP databases for a binary coding and the proposed coding.

(a)

\begin{tabular}{lcc}
\hline & EER & FRR (FAR =0\%) \\
\hline Binary coding & $\mathbf{0 . 0 0} \%$ & $\mathbf{0 . 4 5} \%$ \\
Proposed coding & $\mathbf{0 . 0 0} \%$ & $\mathbf{0 . 0 0} \%$ \\
\hline
\end{tabular}

(b)

\begin{tabular}{lcc}
\hline & EER & FRR (FAR =0\%) \\
\hline Binary coding & $\mathbf{0 . 2 1} \%$ & $\mathbf{1 . 8 1} \%$ \\
Proposed coding & $\mathbf{0 . 0 0} \%$ & $\mathbf{0 . 0 0} \%$ \\
\hline
\end{tabular}

the wavelet packet coefficients and achieves also a good discrimination [34]. Consequently, we now refine the analysis by computing the identification performances obtained for both wavelets, on both ISEP and CASIA databases. Moreover, we look for the best percentage of energy defining the signquantized coefficients (Section 2.4, equation (6)). Figure 12 shows the EER and the FRR $(\mathrm{FAR}=0 \%)$ as a function of this parameter, for both wavelets and both databases. The training set includes the first half of the individuals, and the test set the second half (as described in [24]). Note that the images were manually segmented to avoid misinterpretation due to segmentation errors. The comparison method is global.

The training shows that the most suitable wavelet is the Haar wavelet for both CASIA and ISEP databases. Keeping respectively around $99 \%$ of the energy for defining the threshold $T$ leads to the best performances. These results are confirmed on the test sets. They reinforce the idea that some WP coefficients are more related to noise than to iris texture features and therefore must have less importance in the signature comparison. Table 2 shows the error rates obtained on the complete databases, for the proposed coding (6) compared to the binary coding coupled with a Hamming distance. The performances are significantly improved especially on the ISEP database.

Thus, the proposed signature, extracted from manually segmented images, leads to an identification system without errors. It is worth noting that coding the packet having the third highest energy does not increase the separation between authentic and impostor distributions.

3.2. Robustness Evaluation. We now focus on the robustness evaluation of the identification process, with regards to acquisition conditions. For that, we progressively degrade the quality of the images, by changing the illumination conditions, blurring, and modelling optical axis deviations. All modifications are carried out on the original images. The transformed images are unwrapped according to the parameters provided by the manual segmentation and new signatures are generated. In this way, segmentation inaccuracies do not interfere in the signature robustness analysis. Obviously, this process concerns only images of the test database, the reference signatures being unchanged. Then, the performances are measured for gradually increasing 


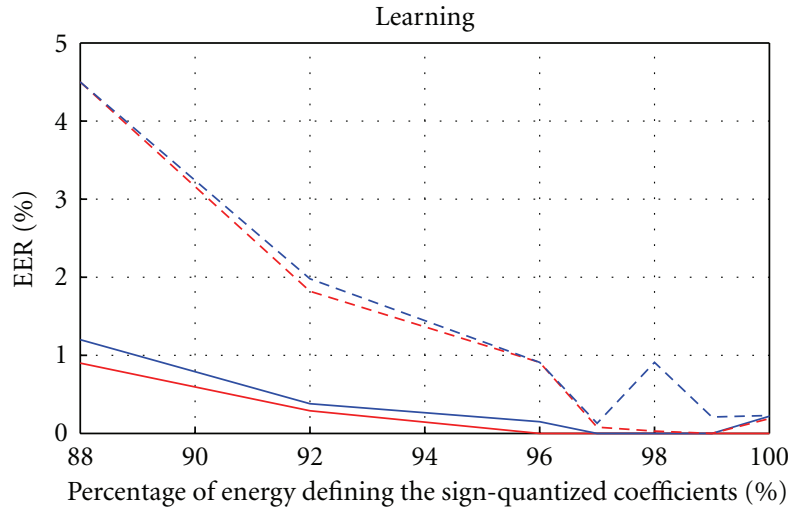

(a)

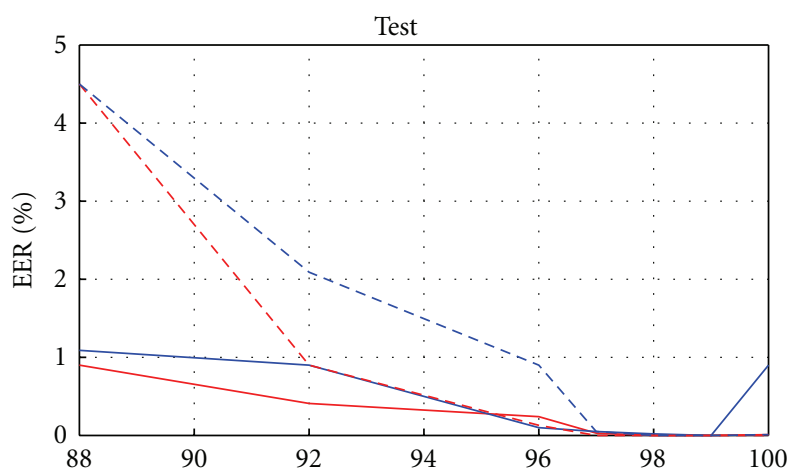

Percentage of energy defining the sign-quantized coefficients (\%)

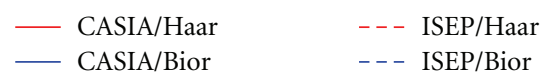

(c)

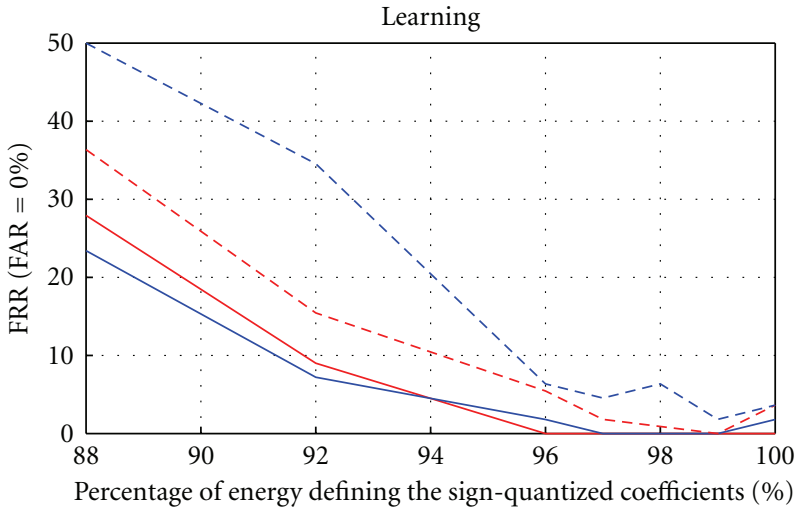

(b)

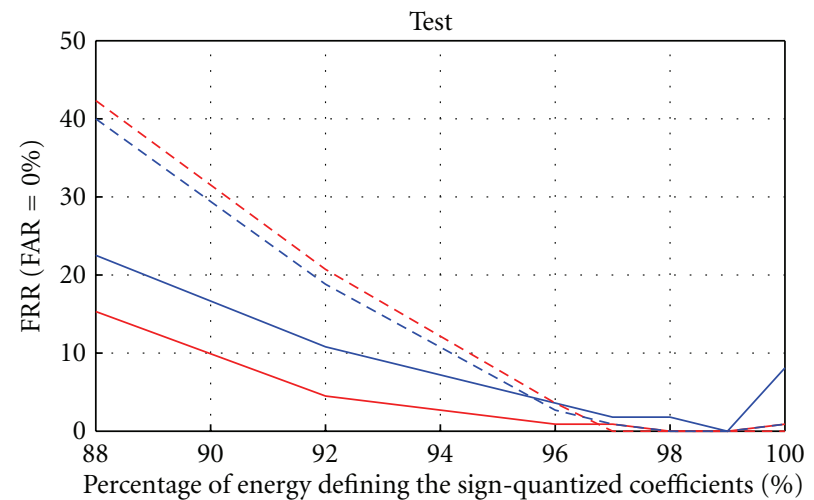

- - - ISEP/Haar

- - - ISEP/Bior

(d)

FIGURE 12: Performance results obtained for the choice of the wavelet and the percentage of energy defining the sign-quantized coefficients. (a) EER and (b) FRR obtained on the training sets; (c) EER and (d) FRR obtained on the test sets.

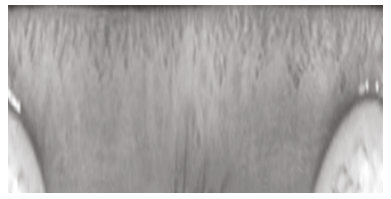

(a)

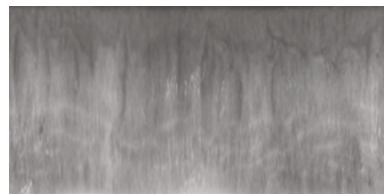

(b)
FIGURE 13: Examples of unwrapped images extracted from (a) ISEP and (b) CASIA databases.

defects. Measurements are done for both global and local approaches. Some visual examples are given in the next subsections for the images represented in Figure 13.

3.2.1. Robustness to Illumination Variations. The tested image, denoted by $I$, is transformed by adding a constant $k$, corresponding to a shift of the grey-level histogram (overexposure or underexposure, Figure 14). The resulting pixel values are limited to the range $[0,1]$ :

$$
I^{\prime}(x, y)=\min (\max (0, I(x, y)+k), 1), \quad I(x, y) \in[0,1] .
$$

Figure 15 shows the EER and the FRR $(\mathrm{FAR}=0 \%)$ as a function of the illumination shift $k$. The performances, obtained with the global comparison method of the iris signatures, show very good robustness to illumination changes, since the EER and FRR remain stable in the range of $k[-0.4,+0.2]$ for the CASIA database (Figures $14(\mathrm{a})$ and $14(\mathrm{~b}))$ and $[0,+0.3]$ for the ISEP database (Figure 14(c)). These results can be linked to the grey-level repartition of the iris texture: the performances start to deteriorate when more than approximately $2.5 \%$ of the pixels are saturated to 0 or 1 . Indeed, the saturation effect leads to the removal of some iris texture, explaining the observed behaviour. On the ISEP database, an underexposure leads quickly to an increase of the error rates, since the database contains at least $10 \%$ of very dark eyes.

The robustness with regard to contrast changes is studied in a similar way, using this time the following transformation

$$
\begin{gathered}
I_{a}(x, y)=a I(x, y), \quad I(x, y) \in[0,1], \\
I^{\prime}(x, y)=\min \left(\max \left(0, I_{a}(x, y)-\bar{I}_{a}+\bar{I}\right), 1\right),
\end{gathered}
$$




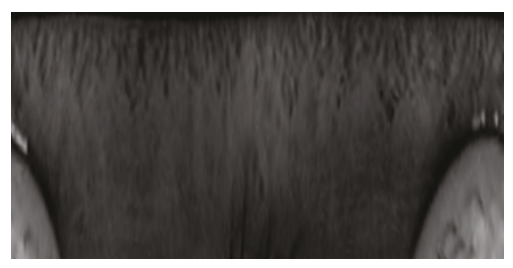

(a) $k=-0.4$

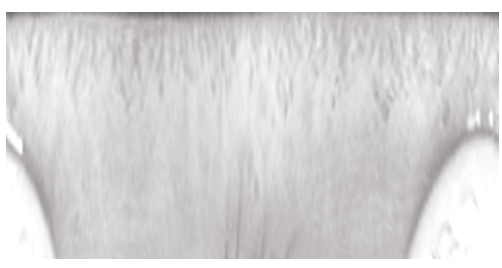

(b) $k=0.2$

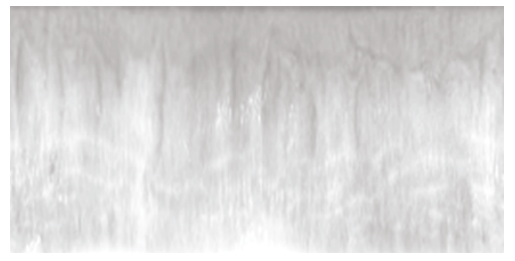

(c) $k=0.3$

FIgURE 14: Examples of illumination variations. (a, b) CASIA image, (c) ISEP image.

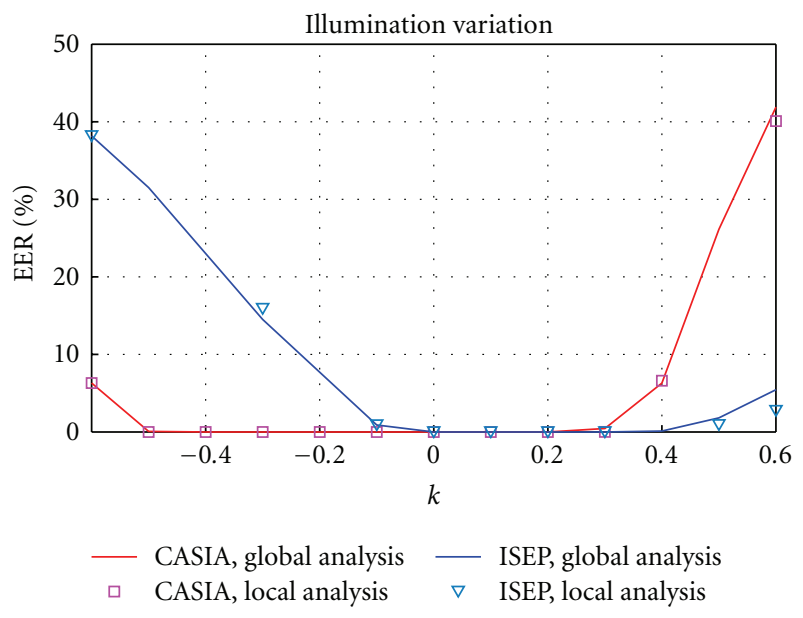

(a)

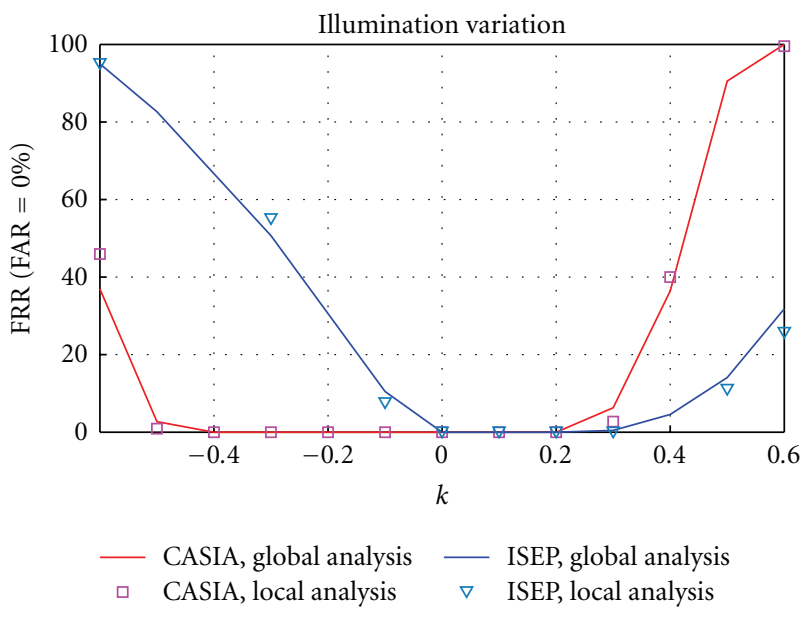

(b)

FIgURE 15: (a) EER and (b) FRR (FAR $=0 \%$ ) as a function of the illumination, measured on CASIA and ISEP databases.

where $\bar{I}$ denotes the mean grey-level of the image. The contrast is decreased when $a$ is lower than 1 and increased otherwise, with a histogram mean unchanged, however with a saturation effect for the darkest and the brightest pixels $(a>1)$ (Figure 16).

Figure 17 shows the EER and the FRR $(\mathrm{FAR}=0 \%)$ as a function of $a$. The admissible loss of contrast is around a factor of 0.1 for the CASIA images and 0.5 for the ISEP images, which demonstrates strong robustness (Figures 16(a) and 16(c)). A high contrast amplification corresponds to some binarization of the unwrapped images (Figures 16(b) and $16(\mathrm{~d}))$. We observe again a very good robustness, since the admissible factors are up to 3 (ISEP) or 5 (CASIA).

A similar robustness to illumination variation has been obtained with the local comparison method.

3.2.2. Robustness to Blurring. The images are blurred with a Gaussian filter of increasing standard deviation $\sigma$, simulating a progressive focus degradation (Figure 18). It is worth noting that the algorithm is very robust, up to a standard deviation equal to $\sigma=3$ (Figures 18 and 19), for the global comparison approach. With the local analysis approach, the EER slightly increases for strong blurring, however insignificantly with respect to the gain obtained in highsecurity mode $($ FRR $($ FAR $=0 \%)$ ).
3.2.3. Robustness to Optical Axis Deviation. Ideally, the images should be acquired with the optical axis orthogonal to the eye. Nevertheless, ocular motion or head rotation movements cause deviations and the image might be nonorthogonally projected on the focal plane, causing deformations of the iris structures. In this study, we simulate vertical and horizontal axis deviations (Figure 20).

The tests show that the algorithm is robust for images with an optical axis deviation up to $15^{\circ}$, in vertical or horizontal direction. The local comparison method reduces both error rates $(\mathrm{EER}, \mathrm{FRR}(\mathrm{FAR}=0 \%))$, since it allows compensating for the distortions induced by the optical axis deviation.

3.2.4. Conclusion. These experiments show that our biometric signature and identification process, applied on accurately segmented images, lead to a zero error identification system, which is furthermore very robust to acquisition conditions, in terms of illumination variability, focusing, and optical axis deviation. These results suggest that restoration techniques, such as deblurring [38], could be useful to improve the segmentation process, but are not necessary for extracting and comparing signatures. As these restoration techniques are heavy and complex, and as they cannot deal with all types image variability (such as variability due to occlusions), 


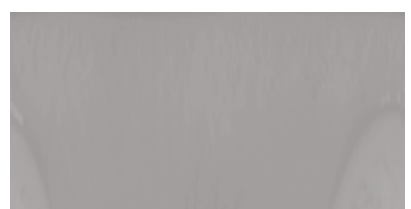

(a) $a=0.1$

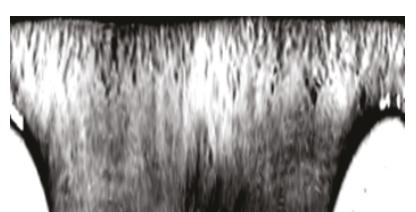

(b) $a=5$

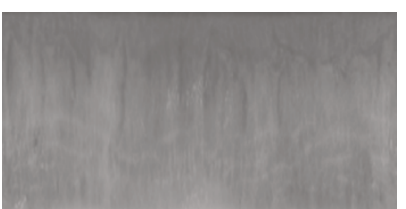

(c) $a=0.5$

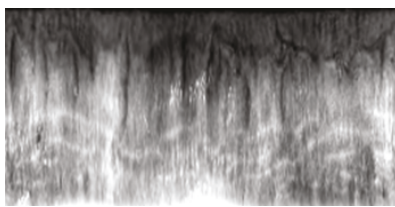

(d) $a=3$

Figure 16: Examples of contrast variations. (a, b) CASIA image, (c, d) ISEP image.

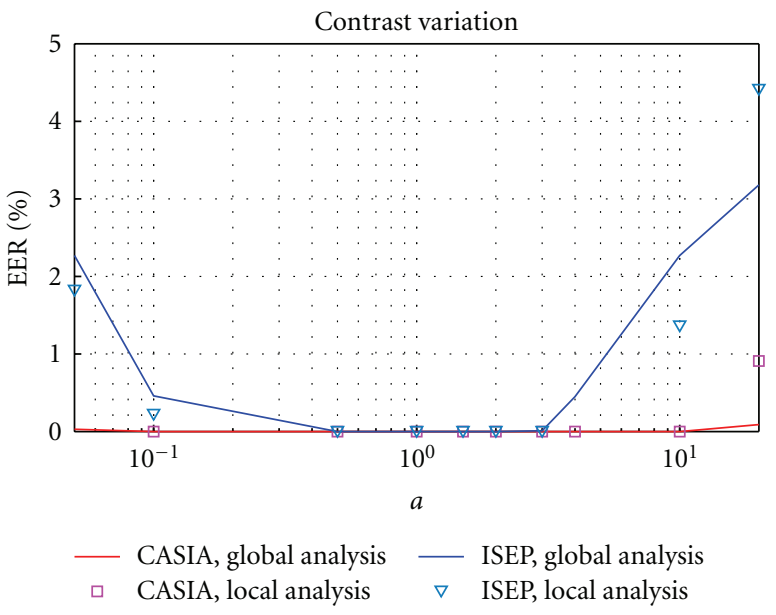

(a)

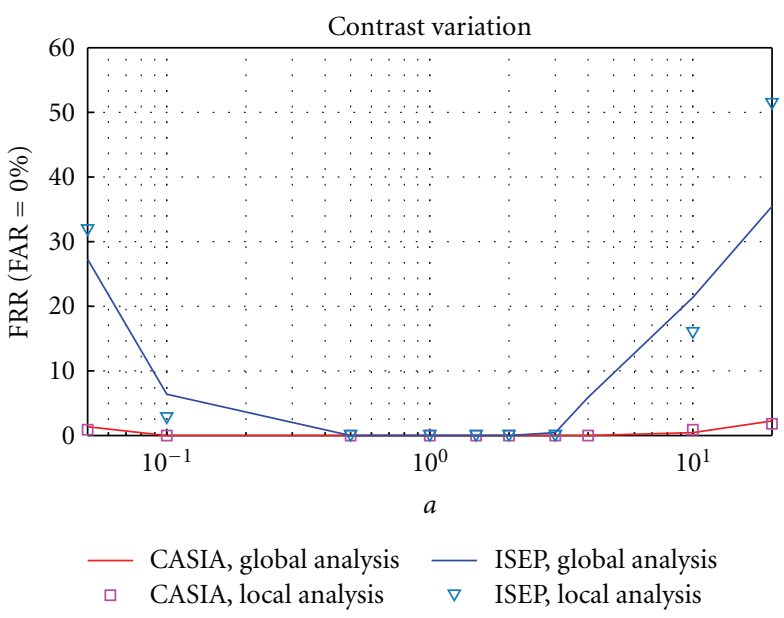

(b)

FIGURE 17: (a) EER and (b) FRR (FAR $=0 \%$ ) as a function of the contrast variation, measured on CASIA and ISEP databases.

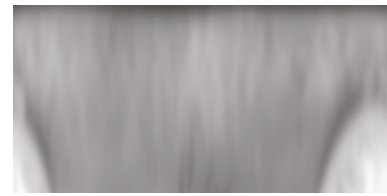

(a)

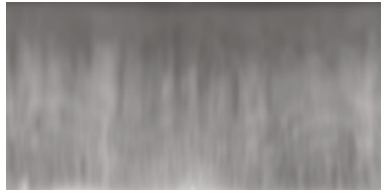

(b)
FIGURE 18: Examples of blurring with $\sigma=3$. (a) CASIA image, (b) ISEP image.

we prefer to address the segmentation imprecision issue at the signature comparison step. In the next section, we will evaluate the benefits of the local analysis method, for dealing with the distortions induced by the segmentation inaccuracies.

\section{Global Performances}

Up to now, the simulations were done on manually segmented images. In the followings, we introduce the segmentation process described in Section 2.3. So, the identification system is fully automatic. Figure 21 shows the performances obtained with both global and local analysis methods. The EER and the FRR $($ FAR $=0 \%)$ are detailed in Table 3 . The $95 \%$ confidence intervals have been calculated using the bootstrap method [39].
TABLE 3: EER and FRR (FAR $=0 \%)$ obtained with automatically segmented databases, for both global and local comparison methods, on (a) CASIA and (b) ISEP databases. Note that the error rates were zero on the manually segmented databases.

(a)

\begin{tabular}{lcc}
\hline & EER & FRR $($ FAR $=0 \%)$ \\
\hline Global & $\mathbf{1 . 8 0} \%[\mathbf{0 . 4 5} \%: \mathbf{3 . 6} \%]$ & $\mathbf{5 . 4 3} \%[\mathbf{2 . 7} \%: \mathbf{8 . 5 6} \%]$ \\
Local & $\mathbf{1 . 3 6} \%[\mathbf{0} \%: \mathbf{3 . 1 5} \%]$ & $\mathbf{2 . 6 4} \%[\mathbf{0 . 4 5} \%: \mathbf{4 . 9 5} \%]$ \\
\hline
\end{tabular}

(b)

\begin{tabular}{lcc}
\hline & EER & FRR $(\mathrm{FAR}=0 \%)$ \\
\hline Global & $\mathbf{0 . 4 7} \%[\mathbf{0} \%: \mathbf{1 . 3 6} \%]$ & $\mathbf{2 . 3} \%[\mathbf{0 . 4 5} \%: \mathbf{4 . 5 2} \%]$ \\
Local & $\mathbf{0 . 4 7} \%[\mathbf{0} \%: \mathbf{1 . 8 2} \%]$ & $\mathbf{1 . 3 4} \%[\mathbf{0} \%: \mathbf{3 . 1 8} \%]$ \\
\hline
\end{tabular}

TABle 4: Performances reached by our local analysis method, compared with the literature.

\begin{tabular}{lcc}
\hline & EER & FRR (FAR =0\%) \\
\hline Daugman's algorithm [40] & $1.44 \%$ & $3.41 \%$ \\
Proença [40] & $1.01 \%$ & $2.39 \%$ \\
Chen et al. [15] & $0.79 \%$ & Non evaluated \\
Proposed & $1.36 \%$ & $2.64 \%$ \\
\hline
\end{tabular}

As expected, there is a performance loss due to segmentation imprecision, but this is significantly reduced by the local comparison, especially in high-security mode. Indeed, 


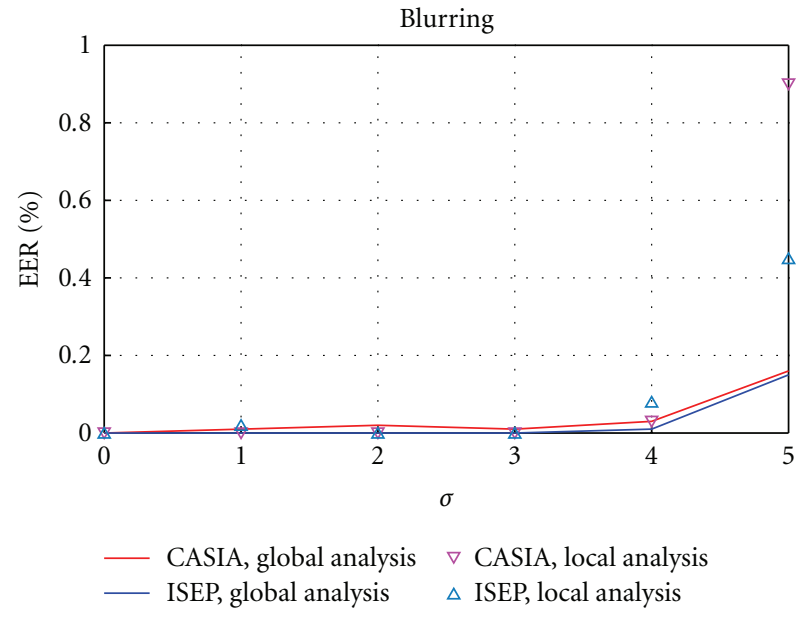

(a)

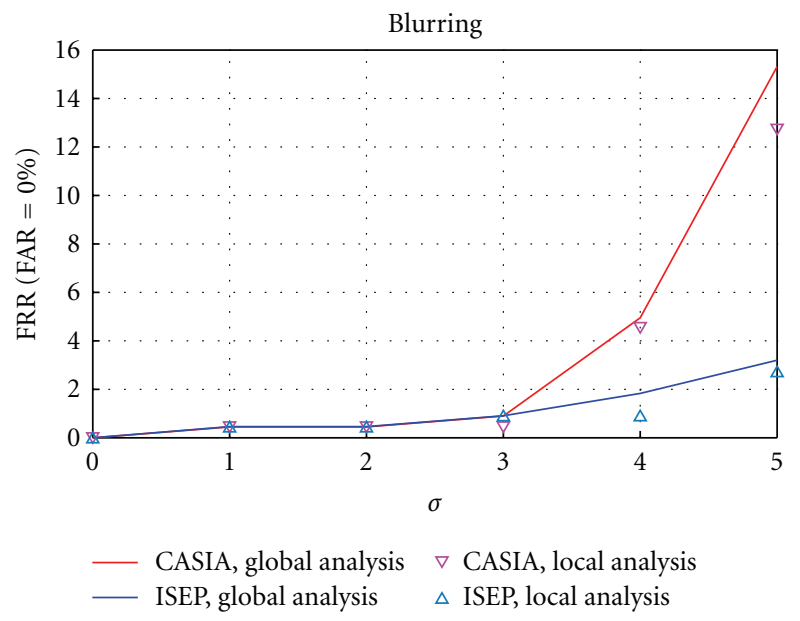

(b)

FIGURE 19: (a) EER and (b) FRR (FAR $=0 \%$ ) as a function of the standard deviation of the blurring Gaussian filter, measured on CASIA and ISEP databases.

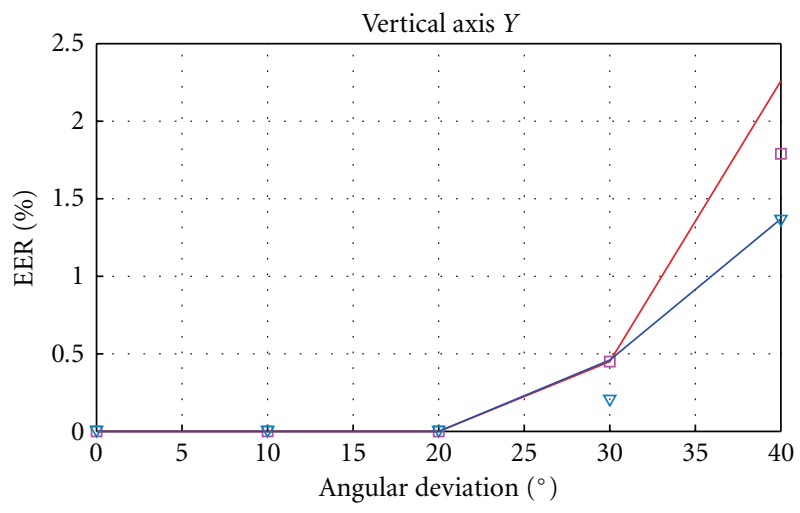

(a)

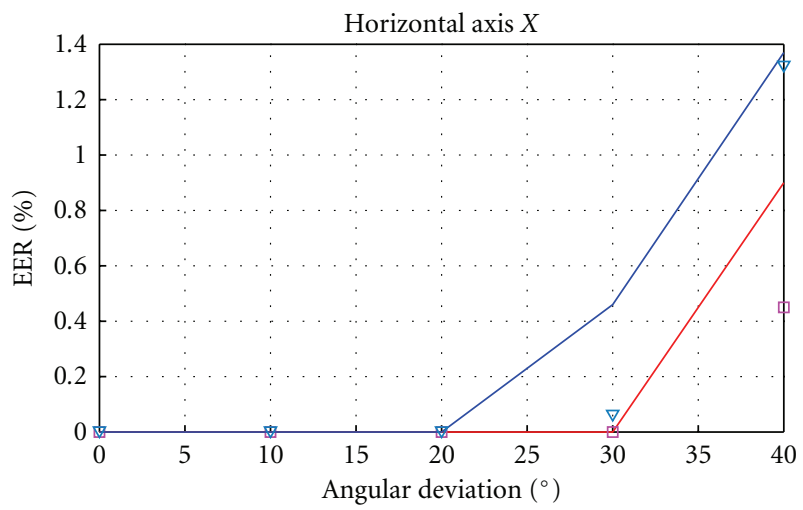

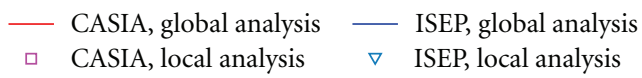

(c)

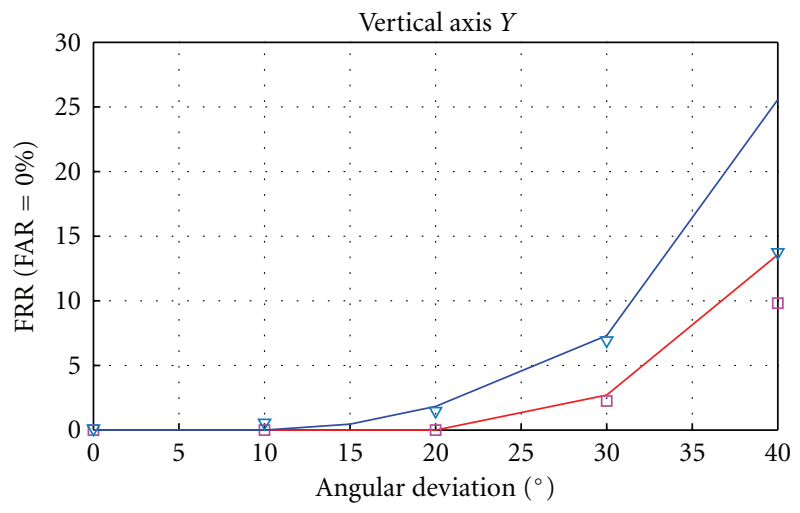

(b)

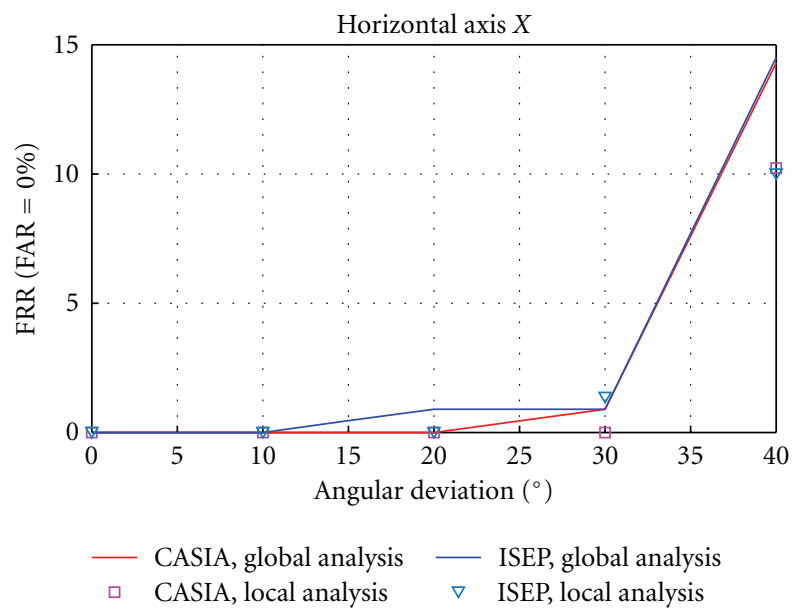

(d)

Figure 20: $(a, c)$ EER and $(b, d)$ FAR $(F R R=0 \%)$ as a function of the optical axis deviation in the vertical $(a, b)$ and horizontal $(c$, d) directions, measured on CASIA and ISEP databases. 


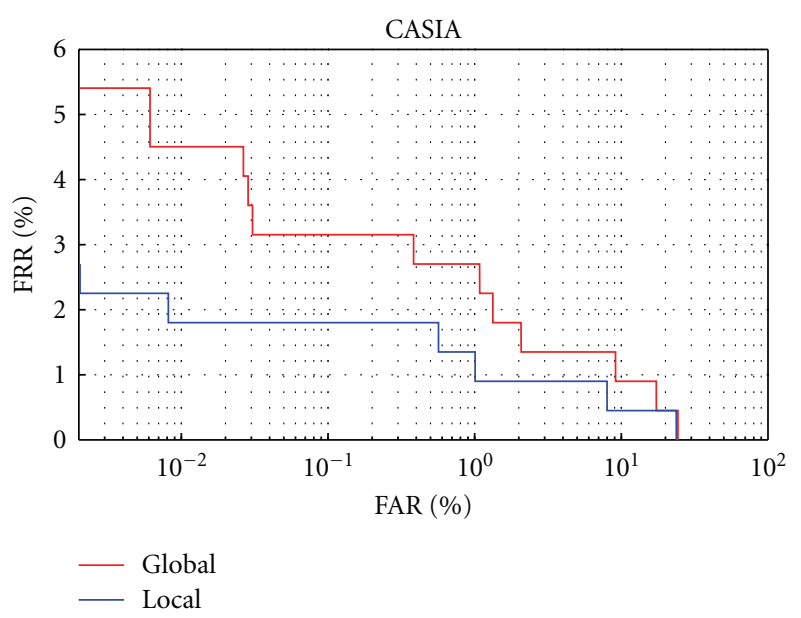

(a)

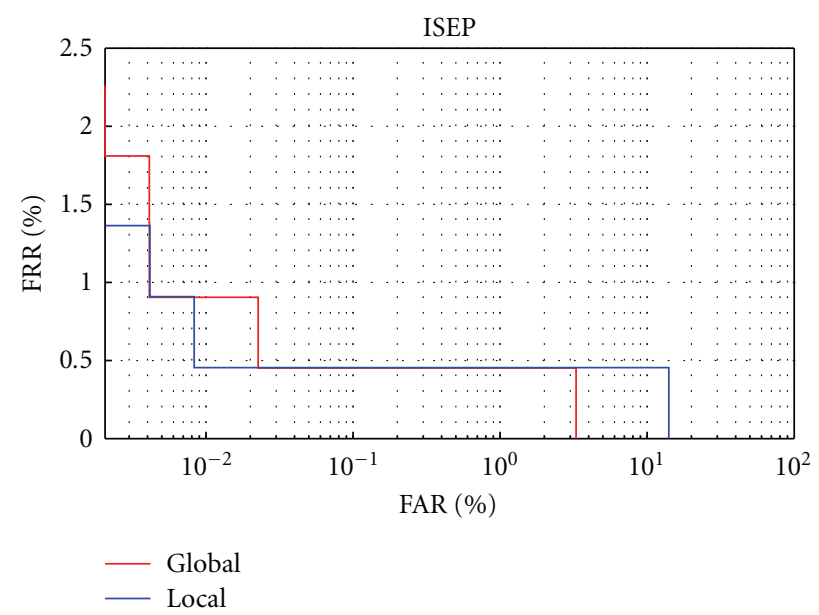

(b)

FIGURE 21: ROC curves obtained with automatically segmented databases, for both global and local comparison methods, on (a) CASIA and (b) ISEP databases.

the local analysis allows compensating for segmentation inaccuracies, as explained in [24]. The results obtained for the ISEP database (Figure 21(b)) confirm the analysis.

Finally, the performances of the fully automatic system, relying on the local analysis method, have been compared with those published in the literature $[15,40]$, on the CASIA databases. In [40], Proença compared Daugman's algorithm [2] with his own approach, on the CASIA-IrisV3-Interval database with a subset of 800 images and 80 subjects. Similarly, Chen et al. [15] evaluated the EER performance on the CASIA V1 database with a subset of 756 images and 108 subjects. In all these experiments, the images were handselected to eliminate incorrectly segmented irises, following a selection procedure similar to that presented in Sections 2.1 and 2.3.4.

As it can be seen in Table 4, we obtain similar results for approximately the same number of iris images (888). However our database contains two or three more subjects (222) and so, these results demonstrate a very good robustness with respect to the increase of subjects.

\section{Conclusion and Perspectives}

In this paper, we presented a ready-to-use iris identification system. Several methods have been proposed and validated for iris segmentation, signature extraction and comparison.

Firstly, a precise and robust pupil and iris segmentation scheme, taking into account eyelid occlusions, is described. The segmentation process is done automatically and its performance is objectively measured. The measurements, realized on two different types of images (visible domain and near infrared), demonstrate that $90 \%$ of the irises are detected with less than $10 \%$ of global error.

A wavelet-packet-based signature extraction method, as well as a novel identification approach, based on the fusion of local distance measures, are further proposed.
Experimental results demonstrate their efficiency in terms of error rates and the benefit of our local-based signature scheme for compensating segmentation imprecision. Moreover, a robustness evaluation of such signature scheme, with regards to the acquisition conditions, has been carried out. The performance comparison, conducted on different iris-databases, highlights the efficiency of our identification step in normal conditions, as well as its robustness to the most common degradation factors: illumination variations, optical axe deviation, and focus degradation. The overall performances of the system could be further increased by improving the accuracy of the automatic segmentation method. Moreover, the presented system could be jointly used with other biometrics approaches (fingerprint, face recognition, etc.) in the design of a multimodal high-security system.

\section{References}

[1] J. Daugman, "Biometric personal identification system based on iris analysis," US patent no. 5291560, March 1994.

[2] J. Daugman, "High confidence visual recognition of persons by a test of statistical independence," IEEE Transactions on Pattern Analysis and Machine Intelligence, vol. 15, no. 11, pp. 1148-1161, 1993.

[3] R. P. Wildes, "Iris recognition: an emerging biometrie technology," Proceedings of the IEEE, vol. 85, no. 9, pp. 1348-1363, 1997.

[4] T. Tambouratzis and M. Masouris, "GA-based iris/sclera boundary detection for biometric iris identification," in Proceeding of the International Conference on Adaptive and Natural Computing Algorithms, pp. 457-466, 2007.

[5] J. Daugman, "New methods in iris recognition," IEEE Transactions on Systems, Man, and Cybernetics, Part B, vol. 37, no. 5, pp. 1167-1175, 2007.

[6] J. Daugman, "How iris recognition works," in Proceedings of the IEEE International Conference on Image Processing, vol. 1, pp. 33-36, 2002. 
[7] W. Boles and B. Boashash, "A human identification technique using images of the Iris and wavelet transform," IEEE Transactions on Signal Processing, vol. 46, no. 4, pp. 1185-1188, 1998.

[8] C. Sanchez-Avila, R. Sanchez-Reillo, and D. De MartinRoche, "Iris-based biometric recognition using dyadic wavelet transform," IEEE Aerospace and Electronic Systems Magazine, vol. 17, no. 10, pp. 3-6, 2002.

[9] L. Ma, "Iris recognition based on multichannel gabor filtering," in Proceeding of the Asian Conference on Computer Vision (ACCV'02), Melbourne, Australia, January 2002.

[10] L. Ma, Y. Wang, and T. Tan, "Iris recognition using circular symmetric filters," in Proceedings of the International Conference on Pattern Recognition, vol. 16, pp. 414-417, 2002.

[11] L. Ma, T. Tan, Y. Wang, and D. Zhang, "Personal identification based on Iris texture analysis," IEEE Transactions on Pattern Analysis and Machine Intelligence, vol. 25, no. 12, pp. 15191533, 2003.

[12] S. Lim, K. Lee, O. Byeon, and T. Kim, "Efficient iris recognition through improvement of feature vector and classifier," ETRI Journal, vol. 23, no. 2, pp. 61-70, 2001.

[13] J. Daugman, "High confidence personal identification by rapid video analysis of the iris texture," in Proceedings of the IEEE International Carnahan Conference on Security Technology, 1992.

[14] J. Daugman, "The importance of being random: statistical principles of iris recognition," Pattern Recognition, vol. 36, no. 2, pp. 279-291, 2003.

[15] Y. Chen, S. C. Dass, and A. K. Jain, "Localized iris image quality using 2-D wavelets," in Proceeding of the International Conference on Biometrics, pp. 373-381, 2006.

[16] Y. Zhu, T. Tan, and Y. Wang, "Biometric personal identification based on iris patterns," in Proceedings of the International Conference on Pattern Recognition, vol. 15, pp. 801-804, 2000.

[17] Z. Ma, M. Qi, H. Kang, S. Wang, and J. Kong, "Iris verification using wavelet moments and neural network," in Proceeding of the International Conference on Life System Modeling and Simulation (LSMS '07), pp. 218-226, 2007.

[18] http://www.milesresearch.com/main/products.htm.

[19] http://www.cbsr.ia.ac.cn/IrisDatabase.htm.

[20] J. Cui, Y. Wang, T. Tan, L. Ma, and Z. Sun, "A fast and robust iris localization method based on texture segmentation," in Proceedings of the Conference on Biometric Technology for Human Identification, vol. 5404, pp. 401-408, 2004.

[21] Z. He, T. Tan, Z. Sun, and X. Qiu, "Robust eyelid, eyelash and shadow localization for iris recognition," in Proceedings of the International Conference on Image Processing (ICIP '08), pp. 265-268, 2008.

[22] M. Adam, F. Rossant, F. Amiel, B. Mikovicova, and T. Ea, "Reliable eyelid localization for iris recognition," in Proceeding of Advanced Concepts for Intelligent Vision Systems (ACIVS '08), pp. 1062-1070, Juan-les-Pins, France, October 2008.

[23] H. Proença and L. A. Alexandre, "A method for the identification of inaccuracies in pupil segmentation," in Proceedings of the 1st International Conference on Availability, Reliability and Security (ARES '06), vol. 1, pp. 224-228, April 2006.

[24] M. Adam, F. Rossant, and B. Mikovicova, "Iris identification based on a local analysis of the iris texture," in Proceedings of the 6th International Symposium on Image and Signal Processing and Analysis (ISPA '09), pp. 523-528, Salzburg, Austria, September 2009.

[25] C.-L. Tisse, L. Martin, L. Torres, and M. Robert, "Person identification technique using human iris recognition," in Proceedings of the International Conference on Vision Interface, Calgary, Canada, May 2002.
[26] T. Randen and J. H. Husøy, "Filtering for texture classification: a comparative study," IEEE Transactions on Pattern Analysis and Machine Intelligence, vol. 21, no. 4, pp. 291-310, 1999.

[27] S. Livens, P. Scheunders, G. Van de Wouwer, and D. Van Dyck, "Wavelets for texture analysis," in Proceeding of IEE Conference on Image Processing and Its Applications, San Diego, Calif, USA, July 1997.

[28] M. Acharyya and M. K. Kundu, "Adaptive basis selection for multi texture segmentation by M-band wavelet packet frames," in Proceeding of IEEE International Conference on Image Processing (ICIP '01), vol. 2, pp. 622-625, October 2001.

[29] N. M. Rajpoot, "Texture classiffication using discriminant wavelet packet subbands," in Proceeding of IEEE Midwest Syposium on Circuits and Systems, August 2002.

[30] R. R. Coifman and M. V. Wickerhauser, "Wavelets and adapted waveform analysis," in Wavelets: Mathematics and Applications, J. J. Benedetto and M. W. Frazier, Eds., pp. 399424, CRC Press, Boca Raton, Fla, USA, 1994.

[31] V. M. Wickerhauser and R. R. Coifman, "Entropy-based algorithms for best basis selection," IEEE Transactions on Information Theory, vol. 38, no. 2, pp. 713-718, 1992.

[32] M. V. Wickerhauser and A. K. Peters, Adapted Wavelet Analysis from Theory to Software, AK Peters, Ltd., Wellesley, Mass, USA, 1994.

[33] A. Laine and J. Fan, "Texture classification by wavelet packet signatures," IEEE Transactions on Pattern Analysis and Machine Intelligence, vol. 15, no. 11, pp. 1186-1191, 1993.

[34] E. Rydgren, T. Ea, F. Amiel, A. Amara, and F. Rossant, "Iris features extraction using wavelet packets," in Proceedings of the International Conference on Image Processing (ICIP '04), vol. 2, pp. 861-864, Singapore, 2004.

[35] L. Deqiang, W. Pedrycz, and N. J. Pizzi, "Fuzzy wavelet packet based feature extraction method and its application to biomedical signal classification," IEEE Transactions on Biomedical Engineering, vol. 52, no. 6, pp. 1132-1139, 2005.

[36] F. Rossant, M. Torres Eslava, T. Ea, F. Amiel, and A. Amara, "Iris identification and robustness evaluation of a wavelet packets based algorithm," in Proceedings of the International Conference on Image Processing (ICIP '05), vol. 3, pp. 257-260, Genova, Italy, 2005.

[37] R. Snelick, U. Uludag, A. Mink, M. Indovina, and A. Jain, "Large-scale evaluation of multimodal biometric authentication using state-of-the-art systems," IEEE Transactions on Pattern Analysis and Machine Intelligence, vol. 27, no. 3, pp. 450-455, 2005.

[38] B. J. Kang and K. R. Park, "Real-time image restoration for iris recognition systems," IEEE Transactions on Systems, Man, and Cybernetics, Part B, vol. 37, no. 6, pp. 1555-1566, 2007.

[39] B. Efron, Introduction to the Bootstrap, chapter 13, Stanley Thornes, Cheltenham, UK, 1994.

[40] H. Proença and L. A. Alexandre, "Toward noncooperative iris recognition: a classification approach using multiple signatures," IEEE Transactions on Pattern Analysis and Machine Intelligence, vol. 29, no. 4, pp. 607-612, 2007. 